\title{
Model-based groupware solution for distributed real-time collaborative 4D planning via teamwork
}

Article

Accepted Version

Zhou, W., Georgakis, P., Heesom, D. and Feng, X. (2012) Model-based groupware solution for distributed real-time collaborative 4D planning via teamwork. Journal of Computing in Civil Engineerin, 26 (5). pp. 597-611. ISSN 1943-5487 doi: https://doi.org/10.1061/(ASCE)CP.1943-5487.0000153 Available at https://centaur.reading.ac.uk/22752/

It is advisable to refer to the publisher's version if you intend to cite from the work. See Guidance on citing.

To link to this article DOI: http://dx.doi.org/10.1061/(ASCE)CP.1943-

5487.0000153

Publisher: American Society of Civil Engineers

All outputs in CentAUR are protected by Intellectual Property Rights law, including copyright law. Copyright and IPR is retained by the creators or other copyright holders. Terms and conditions for use of this material are defined in the End User Agreement.

www.reading.ac.uk/centaur 


\section{CentAUR}

Central Archive at the University of Reading

Reading's research outputs online 


\title{
A model-based groupware solution for distributed real-time
}

\section{collaborative 4D planning via teamwork}

\author{
Wei Zhou ${ }^{1}$; Panagiotis Georgakis ${ }^{2}$; David Heesom ${ }^{3}$; Xiandong Feng ${ }^{4}$
}

\begin{abstract}
Construction planning plays a fundamental role in construction project management that requires team working among planners from a diverse range of disciplines and in geographically dispersed working situations. Model-based four-dimensional (4D) computer-aided design (CAD) groupware, though considered a possible approach to supporting collaborative planning, is still short of effective collaborative mechanisms for teamwork due to methodological, technological and social challenges. Targeting this problem, this paper proposes a model-based groupware solution to enable a group of multidisciplinary planners to perform real-time collaborative 4D planning across the Internet. In the light of the interactive definition method, and its computer-supported collaborative work (CSCW) design analysis, the paper discusses the realization of interactive collaborative mechanisms from software architecture, application mode, and data exchange protocol. These mechanisms have been integrated into a groupware solution, which was validated by a planning team in a truly geographically dispersed condition. Analysis of the validation results revealed that the proposed solution is feasible for real-time collaborative 4D planning to gain a robust construction plan through collaborative teamwork. The realization of this solution triggers further considerations about its enhancement for wider groupware applications.
\end{abstract}

Keywords: 4D CAD; collaborative construction planning; CSCW design; distributed groupware; teamwork

${ }^{1}$ Research Fellow, Design Innovation Research Centre, School of Construction Management and Engineering, University of Reading, Whiteknights PO Box 225, Berkshire, RG6 6AW, United Kingdom (corresponding author). E-mail: w.zhou@reading.ac.uk

2 Senior Lecturer, School of Technology, University of Wolverhampton, Wulfruna Street, Wolverhampton, WV1 1LY, United Kingdom. E-mail: p.georgakis@wlv.ac.uk

${ }^{3}$ Principal Lecturer, School of Technology, University of Wolverhampton, Wulfruna Street, Wolverhampton, WV1 1LY, United Kingdom. E-mail: d.heesom@wlv.ac.uk

${ }^{4}$ Lecturer, School of Design, Writtle College, Chelmsford, Essex, CM1 3RR, United Kingdom. E-mail: xiandong.feng@,writtle.ac.uk 


\section{Introduction}

Extensive studies have discussed four-dimensional (4D) computer-aided design (CAD) topics in the construction field (Koo and Fischer 2000; Li et al. 2003; Dawood et al. 2005; Jongeling and Olofsson 2006; Golparvar-Fard et al. 2009; Kim et al. 2010). Collier and Fischer (1996) initially proposed to link a project plan with its 3D project model for simulating a construction sequence. In such a way, potential conflicts can be discovered before the project delivery. Some industrial projects such as the Walt Disney Concert Hall (Goldstain 2001) have demonstrated benefits adopting this 4D (3D plus time) notion. Following this direction, research efforts have extended to the $\mathrm{nD}$ modeling (University of Salford 2005) by applying advanced building information modeling (BIM) technology. A series of case studies also discussed the effectiveness of BIM applications in the Architecture/Engineering/Construction (AEC) field (Manning and Messner 2008; Khanzode et al, 2008; Pazlar and Turk 2008). Undoubtedly, these investigations are valuable in construction to analyze multifactor relationships among the logical, temporal, and spatial dimensions using information communication technology (ICT) tools. Despite their advancements in cutting-edge applications using 3D models (both CAD and BIM), none of the above examples have discussed model-based groupware for distributed collaboration, which still remains challenges particularly in the fragmented construction field for promoting multidisciplinary teamwork. For the purpose of investigating this area of work, this paper discusses a model-based groupware solution targeting distributed real-time collaborative 4D construction planning.

The construction industry has a multidisciplinary characteristic because of different specialties involved before and during project delivery. 3D CAD/BIM technologies provide subcontractors with possibilities to utilize a unique 3D building model throughout the project for multidisciplinary collaboration (Ku et al. 2008). To enable this multiparty collaboration, synchronous and asynchronous data access/control for the 3D model is essential. BIM solution providers demonstrate different approaches to this functionality in their authoring tools. Autodesk creates worksets in its Revit products such that project team members can work on different parts of a project at the same time (Autodesk 2010), while Bentley uses 'i-model' as a container for open information exchange (Bentley 2011). 
Multidisciplinary collaborators can hence access this to share project information for integrated project delivery. Gehry Technologies adopts the version control system of Subversion in its Digital Project with which project models can be controlled over independent parts for team members' read/write operations (Gtwiki 2009). Tekla Structures applies the master/working model to realize model control in its server/client applications (Tekla 2010). Although these model-based solutions allow multiple users to concentrate on the 3D building model for collaboration, they merely aim at the low level of model data integration and sharing. Beyond this data level's collaboration, limited features are available from them to promote multidisciplinary collaborators' teamwork, which is more concerned about human issues particularly in the distributed context (Grudin 1994; Shelbourn et al. 2007).

Construction project management calls for effective teamwork and synergy (Uher and Loosemore 2004) whilst construction planning plays a fundamental role in project management (Hendrickson 1998). However, a significant phenomenon in construction is that multidisciplinary subcontractors of a project are often geographically dispersed before on-site work starts. Taking construction planning into account, multidisciplinary planners in a planning team usually focus on their own specialty work in different places. Their independent planning work inevitably causes conflicts when integrated for the project delivery (Kang et al. 2007; Zhou et al. 2009). For overcoming this kind of teamwork obstacle, 4D technology is highlighted for collaborative construction planning (Heesom and Mahdjoubi 2004). An empirical study (Kang et al. 2007) shows that 4D simulation across the Internet can help detect more conflicts in a construction plan. Thus networked collaborative 4D CAD can benefit geographically dispersed planners' work. The collaborative approach applying 4D is also envisaged to improve other knowledge based work, e.g. 4D-BIM construction safety planning (Sulankivi et al. 2010). Based on these initiatives, it is evident that a model-based 4D-planning groupware can support a group of geographically dispersed planners to achieve a robust construction plan through collaborative teamwork. Nevertheless, the realization of this groupware has three challenges in methodological, technological and social aspects.

The methodological challenge is to properly address the relationship between 4D modeling and planning in a networked environment. Since the initiation of 4D technology over a decade ago, almost all studies and application solutions target stand-alone systems for individual planners. Their 
typical methods of 4D CAD creation are to manually link an inputted 3D model with a developed project plan. In such a way, elements of the 3D model - the product breakdown structure (PBS) can connect with plan tasks - the work breakdown structure (WBS). 4D dynamic sequence simulation then can be generated to review the plan using some third party toolkits such as Autodesk Navisworks, Bentley ProjectWise Navigator, etc. This 4D CAD creation method needs separate tools for 3D modeling, planning and 4D simulation. Despite adopting a 4D CAD functionality into 3D modeling environments, commercially available BIM authoring tools, such as Tekla, Digital Project and others, still require the user to input developed plan data to generate 4D CAD. Such a fragmented working process is obviously inconvenient and ineffective, particularly in networked situations to integrate various plan data from different planners. Targeting this problem, Sriprasert and Dawood (2003) created the multi-constraint planning system that integrates plan data via a central database. The system enables multiple users to access the database from the web and mobile platforms to remotely operate plan data. Nonetheless, it applies the typical 4D CAD creation method to connect the developed plan database with the inputted 3D model. This methodological limitation makes the system a collaborative review system where multiple users can examine the developed plan being presented by the 4D CAD, rather than a collaborative planning system where multiple users can engage in planning. Integrating 4D modeling into the planning process could be a viable approach to networked collaborative planning for the 4D groupware creation.

Integrating 3D planning and 4D simulation gives rise to the technological challenge in the network condition. Related studies show the possibility of using 3D CAD model directly for planning (Frohlich 1997; Vries et al. 2007) and BIM model for 5D (4D plus cost) modeling (Tanyer and Aouad 2005 ) in the stand-alone situation. These research achievements invoke a further consideration that collaborative 4D construction planning could be achieved by applying a 3D model underpinned by the network infrastructure. Networked multidisciplinary planners can thus concentrate on a unique 3D model to collaboratively perform construction planning, and generate 4D simulation to examine the robustness of the plan during the planning. To satisfy these needs, the $4 \mathrm{D}$ groupware should own the features of identifying and handling different data types, which come from multiple users when they perform concurrent planning and take collaborative actions across the Internet. Synchro 4D as a commercial application demonstrates its capability for concurrent planning. It uses a server/client 
software architecture where the client application enables individual planners to perform 3D planning and 4D modeling while the server application can integrate all plan data from clients (Synchro 2010). Similar to the model-based BIM authoring tools discussed above, Synchro 4D only enables the user to update and integrate plans at a data level. This system limitation hampers users from gaining sufficient information with each other, and thus it is unlikely to turn their individual concurrent planning and 4D modeling into collaborative teamwork.

Social dynamics brings challenges for collaborative teamwork in groupware development (Grudin 1994). 4D groupware creation is particularly about supporting users' tasks, behaviors and their social activities as teamwork for problem solving in a shared 3D context. Zhou et al. (2009) highlighted three levels of collaboration in the interactive definition method to deliver collaborative 4D planning via teamwork. At a low level, generated plan and simulation data from multiple users can be integrated similarly to Synchro 4D. At middle and high levels, multidisciplinary planners can use computer systems to communicate and interact with each other directly and indirectly. In such a way, they can organize and coordinate their individuals' tasks and behaviors in the networked environment. This kind of computer-supported collaborative work (CSCW) is essential to foster and motivate effective collaboration across multidisciplinary boundaries to be interdisciplinary work (Fischer et al, 2005). Fruchter (1999) highlighted that computer supported collaborative teamwork needs to be integrated into system development to enhance networked information exchange and communication. Due to these $\mathrm{CSCW}$ requirements from teamwork, the 4D groupware thus needs to provide a set of mechanisms to satisfy team-based collaborative features and functionalities in analyzing a 3D model for planning strategy exploration, coordinating planners' communications and actions, etc. Nevertheless, a survey shows that CSCW in the construction industry does not get deeper from the current information-rich multidisciplinary work to the more creative interdisciplinary work (Garner 2003). The majority of popular model-based 3D/4D CAD/BIM solutions are usually short of related collaborative mechanisms to support intimate teamwork. This disadvantage hampers the creation of groupware solutions for more creative applications.

The aim of this paper is to discuss a model-based groupware solution for distributed real-time collaborative 4D construction planning. It can enable multidisciplinary planners to focus on a unique 
3D model to perform interdisciplinary collaborative planning across the boundaries of time, space, and specialty. The innovation of this groupware solution lies in its collaborative mechanisms to handle multilevel data for interactive and collaborative teamwork. Combining both social and technical considerations, the paper firstly discusses the method and its underlying CSCW design of the interactive collaboration in 4D construction planning. On the basis of this discussion, it further proposes the groupware solution to disclose its collaborative mechanisms in terms of software architecture, application mode and data flow, as well as data exchange protocol. This is accompanied by the implemented prototype named 4DX and its application features. Furthermore, the paper delineates the validation of the proposed solution conducted by a planning team in a truly geographically distributed condition. Some unveiled insights in the validation contribute to the discussion of further enhancement of the solution.

\section{Method of Collaborative 4D Construction Planning}

Collaborative 4D construction planning asks for multidisciplinary planners to perform, as a team, planning work based on the network infrastructure. It needs contributions from individual creativity and social creativity (Fischer et al. 2005). In order to achieve this aim, the interactive definition method was created to involve individual planners for a real-time collaboration (Zhou et al. 2009). The method emphasizes an open shared social-technical context, social interaction, user-system interaction and plan data incorporation. This section highlights the essence of 4D interactive collaboration in the interactive definition method and further clarifies the principle of CSCW design in the method.

\section{Interactive Collaboration in 4D Planning}

Collaborative 4D planning is a correlated social-technical activity. Its correlation includes three levels of interaction in a social-technical context, in which inputting a unique 3D model is essential to achieve this aim. Within such a context, social interaction among collaborators is available via human-human communication at a high level. Multidisciplinary planners can communicate with each other to discuss planning strategies and formulate planning solutions. User-system interaction, at a 
middle level, enables planners to interactively explore the 3D model and perform planning via artifacts in their local systems. It can also promote planners' social interaction at this level. In view of the multidisciplinary collaborative nature in this multiparty planning, a proper CSCW design can ensure planners' work is open-ended and motivated. Based on the on-line connectivity, created plan tasks by different planners are incorporated to be a complete plan at a low level. 4D simulation, as a plan conflict detector, is generated during plan task definition. Combining these aspects, distributed collaborative planning can lead to a synthesized plan and 4D simulation (Figure 1).

In order to realize this collaborative planning, inputting a 3D model (either entity-based or BIM-based) can foster both social interaction and user-system interaction for effective teamwork. This unified 3D model permits access to all planners such that they obtain their own building information, namely PBS, through 3D navigation and direct manipulation. These operations include zoom in/out, pan, rotate, decompose, and pick up/off, etc. in a 3D environment. Planners can thus define associated tasks on related 3D elements, and further generate 4D simulation. In the interactive definition method, a set of established parameters called simulation (SIMU) items can help planners define plan tasks and related simulation on 3D elements. This method is thus named PBS-create-WBS (PBS $\rightarrow$ WBS). Its feasibility has been verified in a local area network (LAN) situation (Zhou et al. 2009), in which related CSCW design in the method plays an irreplaceable role in the collaboration process.

\section{D CSCW Design}

Greenberg (1991) stated that "CSCW is about how people work together, and how the computer and related technologies affect group behavior." For the purpose of effectively supporting geographically distributed planners for collaborative 4D planning, any related CSCW design ought to facilitate the planners' mental process, social interaction and task performance. It strives to make their planning process open-ended, synchronous and communicated through teamwork. According to the enumerated features of communication, configuration, coordination, information access, interaction, and usability in CSCW design (Mills 2003), the interactive definition method involves a series of collaborative teamwork sessions in its application processes. Individual planners hence can team up for 
co-creating a robust plan across the Internet. These sessions are co-navigate, co-sort, co-plan, cosimulate and co-talk (Zhou et al. 2009).

Co-navigate and co-sort sessions are dedicated to analyzing spatial structure of a 3D building model. Planners can co-navigate and analyze the 3D model together via zoom, pan, rotate, and decompose to identify suitable 3D elements for defining plan tasks. The co-sort session enables planners to interactively pick out related PBS items together from the 3D model whilst the co-plan allows them to define simulation items on the co-sorted 3D elements for concurrent planning. The defined items are then broadcast to every online planner and synthesized to be a complete plan. In conjunction with online planners, co-simulate can help detect potential conflicts in the generated plan across the network. Involved online planners can check potential conflicts, not only in their own, but also the overall plan during the co-simulate session. In order to maintain a live social interaction in planning, the co-talk session is available for this purpose. Audio-video conferencing and text chatting are applicable choices in the session.

These collaborative sessions have two types. One is a conditional session, which requires an invitation-acceptance agreement among collaborators. Co-navigate, co-sort, and co-simulate belong to this type. The conditional session has a specific lifecycle about creation, process, and termination. Its creation is dependent on the session holder and potential session attendees. The session holder is an online collaborator who creates a collaborative session by sending out an invitation. Other online collaborators are potential session attendees. Once accepting an invitation from a session holder, they become the session attendees and a live conditional session is then created for teamwork. During a live session through the processes of co-navigate, co-sort or co-simulate, the involved attendees and the session holder can collaborate together for planning work. The session holder plays a dominant role in controlling the session's progress. Both the session holder and the attendees can perform related concurrent operations, which are synchronously reflected in their systems. A live session can be terminated at any time by either the session holder or the session attendees. Another session type is an unconditional session, which can be launched by any online collaborators at any situation. The co-talk session is a kind of unconditional session for communication using text chatting. The characteristic of the unconditional session is that online planners can conduct them freely and concurrently. It has no 
specific lifecycle about creation and termination when applying it. The co-plan session can be either conditional or unconditional session depending on collaborators' needs.

Conditional and unconditional sessions have different features. The conditional session is close-ended that its collaborators are unable to join in another live conditional session at the same time. A live conditional session has no influence on other sessions and on non-invited collaborators, who also have no permission to join a created live session. Some synchronous operations and controlled data of the session, e.g. zoom, pan, rotate etc. are limited within the session holder and the attendees. The unconditional session, on the other hand, is open-ended that online planners can collaborate with each other whether they are in a session or not. In other words, once planners are connected with the network, they can perform unconditional sessions of text chatting and co-plan without constraints. It is possible that several conditional sessions co-exist during planning. In such a scenario, an unconditional session is a bridge to connect those parallel sessions and non-session planners for information exchange (Figure 2). These features provide planners with flexible choices in collaboration. Depending on specific planning situations, planners can choose related partners to create suitable conditional sessions for focus group collaboration. Thus the whole collaborative teamwork in planning can be organized dynamically and effectively.

\section{Proposed Solution}

In the light of foregoing discussion on 4D interactive collaboration and related CSCW design, the distributed 4D groupware ought to offer certain functionalities to underpin related social-technical activities to achieve collaborative planning. These functionalities are helpful to clarify the groupware solution from three aspects: software architecture, application mode and data flow, as well as data exchange protocol. The software architecture includes the main functional modules and components of the system to support collaborative activities. It also decides its application mode and data flow when performing those activities. In the meantime, stipulating a set of data exchange protocols is necessary for realizing effective data transmission in real time. In view of the current dominant situation of entitybased 3D CAD model in the industry (Tse et al. 2005), the proposed 4D groupware targets non-BIM based 3D models to discuss its development in a more generic condition. 


\section{Software Architecture}

The proposed software architecture consists of a client tier and a server tier (Figure 3). These two tiers own the modules of 4D builder and 4D item pool respectively. Both tiers also contain a module of communication services with related management components. Among these modules, the 4D builder in the client tier includes two components of 3D element container and SIMU item container. They can provide PBS information for simulation item generation and restoration. The 4D builder also involves three components including file management, plan browser and 4D player for those containers' management and utilization. In the server tier, the 4D item pool module owns three components of 3D element container, SIMU item synthesizer, and role-attribute reactor to provide collaborative information to the client tier. It links several components of browsers and file management. These four fundamental modules are all connected with an event hub module in two tiers for data transmission.

\section{Event Hub}

The event hub module, being included in both the server tier and client tier, takes responsibility for exchanging data between two tiers. It has an event-based mechanism for identifying, sending, and receiving information to ensure effective data exchange. In order to achieve this aim, the event hub contains a set of components including event enumerator, event identifier, data carrier, and exchange centre (Figure. 4). The event enumerator defines a series of system events, which can deal with any data operations and updates from both system and users. They help describe specific requests for data sending or receiving internally and externally, and then choose corresponding operations. The event identifier functions by getting an incoming event internally or externally, and interprets it according to event definition by the event enumerator. As a result, the data carrier is triggered to choose a suitable data buffer for loading the coming data. These data then are passed to either the exchange centre for external transferring or internal components directly. Both event hubs in the client tier and server tier adopt the same event-based mechanism. The only difference lies in their converse operations for data exchange, e.g. an event can result in sending operation in the client tier whilst the same event can lead to receiving operation in the server tier. 


\section{Communication Services}

Both the client and server tiers contain a communication services module. In the server tier, the module has the component of role services to provide definition of specialty role for collaborators. Considering collaborative planning from multidisciplinary planners, collaborators need to explicitly decide their expertise in the planning process. The role services component in the server tier can record collaborators' role choices and their states in collaborative processes. The role definition is managed by the communication management component, which allows input/output or directly edit operation to manage specialty roles. Besides these role services, the communication services module is also in charge of server start or stop as well as data dispatching among collaborators. Underpinned by the event hub, the communication services module can identify upcoming events' sources and destinations, and thus dispatch carried data to the relevant collaborators.

In the client tier, the communication services module consists of the components of the role register and the messenger. According to the user's specialty, the role register component enables collaborators to select a state and a role provided from the role services component in the server tier. It is also used for recording and displaying other collaborators' states. On the other hand, the messenger can support collaborators' communication through text chatting. By receiving data from the server tier, the messenger displays incoming texts to local planners. It also ensures collaborators input text and send to targeted online planners via the server. Additionally, the conference centre component can host other advanced built-in components for audio-video conferencing if needed. Besides this option, commercially available audio and videoconferencing services are applicable for concurrent engineering as they can directly contribute value to team efforts (Pena-Mora 2000).

\section{D Builder}

The 4D builder module in the client tier can synthesize simulation items with 3D model elements. Its SIMU item container component contains a series of text-based task items to record defined WBS items and their associated PBS elements. Another component of the 3D element 
container possesses a series of 3D graphical elements to represent inputted PBS. Because a simulation item wraps PBS elements with WBS item together, the SIMU item container and the 3D element container are interrelated with each other. Every item in the SIMU item container can refer to its related $3 \mathrm{D}$ elements in the 3D element container, and vice versa. In the offline condition, these two containers can be managed by their file systems to input and/or output 3D model files and simulation item files respectively. In the online condition, the containers can be filled in through retrieving data from the 4D item pool in the server tier. Additionally, both of them in the client tier have a browser to view their contents. The browser of the $3 \mathrm{D}$ element container is also a $4 \mathrm{D}$ player. It can not only assist $3 \mathrm{D}$ model analyzing and viewing, but also present 4D simulation when defined simulation items are synthesized. The browser of the SIMU item container can help view defined simulation items and hierarchal plan structure, which is generated by a plan editor. The planner can define simulation items via this editor by inputting appropriate values

\section{D Item Pool}

The 4D item pool module in the server tier has three components of $3 \mathrm{D}$ element container, role-attribute reactor, and SIMU item synthesizer. As a central data repository, these three components in this module can supply the client tier with unique 3D elements and their attributes, as well as synthesized simulation items. All of them provide content management through their own file systems, and each of them owns a browser for checking its content.

The applicable 3D model for this module adopts Autodesk .3DS and Microsoft .X formats. A simple 3D model browser can be used for checking a loaded 3D model in the 3D element container. It displays an overall 3D model profile for the administrator's management. Specific spatial information of the loaded 3D elements is unnecessary to show in the server tier. Nevertheless, their names need to be listed in the role-attribute browser to build correspondence between defined specialty roles and the 3D elements. This correspondence will help decide each 3D element's attribute for every defined role. The planners can thus perform simulation item definition according to generated 3D elements' attributes. The SIMU item synthesizer is applied for integrating defined simulation items from different planners. According to every defined item's specification and overall plan situations, the synthesizer 
will assign each item with a unique order to generate an integrated plan. Its browser can be applied for viewing the generated hierarchal plan.

The role-attribute reactor takes the responsibility of generating 3D elements' attributes - the PBS elements' attributes for each collaborator. It also deals with PBS read/write access control. As collaborators focus on their own PBS elements for task definition, the reactor can thus lead to such a result that only writable PBS elements of a collaborator can be defined with tasks. It means that collaborators ought to decide PBS elements' read-write privilege beforehand so as to suit their planning specialties. Such a mechanism was described as user's perspective in the research of distributed virtual engineering (DVE) (Maxfield 1998). It applied an information mask, namely filter, to get meaningful information for different clients. A similar concept can be applicable for model-based collaborative 4D planning to identify planners' interested information. In case an inputted 3D model owns building information, e.g. BIM model, its PBS items' read-write privilege for collaborators can be decided simply according to its internal differentiation. However, an inputted 3D model without building information requires a mechanism to identify PBS, and then decide its privilege for collaborators. In the proposed groupware, visual identification is a feasible way to realize this aim. Given a 3D element without building information, the role-attribute reactor ought to consider four possible visual identification results for attribute generation: Free (F), Readable (R), Writable (W), and Conflict (C). A specific element attribute value is dependent on all collaborators' selection results. This interrelated situation is illustrated in Table 1.

Situation 1 in Table 1 shows a PBS element is of free attribute for all collaborators. It can be selected by the collaborators to set the writable attribute if the element belongs to their domains. Once a PBS element - a 3D element is set with the writable attribute by only one collaborator e.g. collaborator A, then this element's attribute becomes readable for other collaborators. This condition is corresponding to the situation 2 in the table. In case a writable element is further picked out by another or more collaborators, this element then is in a conflict state. Its attribute is a conflict for those coowners but not other collaborators who do not select the element. It still has a readable attribute for them. This condition is corresponding to the situation 3 in the table. Given an element with the conflict attribute, co-owners could give up their selections so that the element can be of either writable attribute 
for only one owner, or free attribute for all collaborators. This role-attribute reaction mechanism can dynamically generate a PBS element's attribute for every collaborator depending on all collaborators' selections. Its specific attribute is different from role to role. The administrator can monitor this assignment through the role-attribute browser.

\section{Application Mode and Dataflow}

The proposed distributed software architecture is based on the server/client paradigm. The server is a central data repository connecting with a series of clients (Figure 5). The connection between the server and the clients can be through the Internet or LAN depending on collaborative requirements. In view of adopting the file management system in the server side, one of clients, possibly the main contractor, needs to be located in the same place with the server. Besides a planner's role in collaborative planning, the user of this client ought to play an administrator's role in maintaining the server's normal working.

The shared repository structure of the server/client mode implies that any collaborative work among clients needs the server's link. Involved data transmission between the server and the clients follows three approaches of updated broadcast, direct transfer, and automatic retrieve (Figure 6). The updated broadcast and the direct transfer follow several procedures. Firstly, an operation is performed via a user interface of $4 \mathrm{D}$ builder or communication services in client $\mathrm{A}$. The conducted operation then carries some data from the 4D builder or communication services to the event hub. Secondly, it triggers its registered event to be dealt with by the event hub, and delivers the event and data via the network to the event hub of the server side. Afterwards, the server side can parse the transferred event and choose corresponding operations. These operations result in either updated broadcast or direct transfer to client B. The former causes data updating in the $4 \mathrm{D}$ item pool and broadcast to all clients whilst the latter causes data to be dispatched directly to target clients. Additionally, the automatic retrieve simply occurs when a client connects the server to get information from the 4D item pool. Its data flow is partly the same with that of the updated broadcast. The difference is that retrieved data are only transferred to the requested client but not to be broadcasted to other clients. 


\section{Real-Time Data Exchange Protocol}

Given three data transfer approaches between the server and the client, establishing a set of protocols can help the system choose suitable approaches to effective data exchange in real time. These protocols decide transferred data to follow right approaches and reach correct destinations. The protocols include automatic data flood and controlled data transfer. The former further consists of static data flood protocol and dynamic data flood protocol.

The static data flood protocol has an effect on retrieving existing data directly from the 4D item pool of the server to the clients. Existing data, including the PBS elements, their attributes, defined roles, and generated simulation items, are usually recorded and stored in the server side. When the user connects the server to log in, these data will be automatically retrieved from the server to the client. This static data transfer protocol enables users to obtain existing data from the server following the automatic retrieve approach. Its transfer direction is singular from the server to the clients.

The dynamic data flood protocol, on the other hand, follows the approach of the updated broadcast in order to transfer new generated information to the server for restore and synthesis, and further to broadcast to all online clients. This protocol takes effect during the user's log in/off the server. When logging in the server, the user needs to input a user name, select a role and a working state. This new information is sent to and recorded in the server, and broadcasted to other online users. Similarly, when a user logs off the server, other online users are informed instantly. During collaboration periods, moreover, newly updated PBS attributes and defined simulation items by a client also apply this protocol to broadcast them to all clients. Its transfer direction is multiple for all online clients. Both static and dynamic data transfer protocols maintain a unified real-time environment whenever collaborators join in, leave, and participate in collaborative work.

The controlled data transfer protocol works in the collaborative sessions of co-navigate, cotalk, and co-simulate. A significant feature of this protocol is that exchanged data are assisting information without the needs of restore and display in the server. A session holder can control the session's creation, termination, process, and contents whilst session attendees are passive to receive 
these data. It has no influence on other online collaborators who are out of the session. Its data flow adopts the approach of direct transfer from the session holder to the session attendees. Therefore, its data transfer direction is limited within several related clients only.

Nevertheless, the controlled data transfer protocol is usually overlapped with the automatic data flood protocol in the collaborative sessions of co-sort and co-plan. In view of overhead data such as simulation items and PBS attributes generated in these sessions, the automatic data flood protocol is applicable to broadcast these data to all online collaborators no matter they are in or out of collaborative sessions.

\section{Implemented Prototype}

In accordance with the rationale of the proposed solution, a prototype named 4DX was implemented at the University of Wolverhampton in the UK. Its development toolkits are Microsoft Visual C++ 8.0 (Visual Studio 2005), Direct3D 9.0, and Winsock 2.0. They are popular utilities for the Windows platform to create networked real-time 3D graphical applications, and compatible with most hardware. The object-oriented programming (OOP) approach is applied for the system development. The prototype consists of a server side application and a client side application running in the network condition. Its underlying communication is based on the TCP/IP protocol. The implementation results are presented as follows.

\section{Server Side Application}

The server side application constructs the server tier's modules of event hub, communication services, and 4D item pool. It integrates necessary collaborative planning information in the 4D item pool for retrieving and updating from client sides. This collaborative planning information includes 3D elements and their attributes, simulation items, as well as specialty roles. The management of this information is performed through separate file systems of the components of $3 \mathrm{D}$ element container, role-attribute reactor, SIMU item synthesizer, and role editor respectively. Each of these components provides a browser integrated in the user interface for content checking and managing (Figure 7). 
A thumbnail 3D model browser is dedicated to the 3D element container. It provides basic 3D navigation operations such as zoom, pan, and rotate for simply browsing a loaded 3D model. 3D elements' names are displayed in the browser of the role-attribute reactor when a 3D model is loaded in the server. The role-attribute browser can combine defined specialty roles to show the attribute of each 3D element while collaborators decide PBS items' read-write privileges in their clients. The definition of specialty role is through the role editor. Each defined role with its user name and working state is displayed in the role browser. Moreover, the browser of the SIMU item synthesizer displays a synthesized result of simulation items in a text-based hierarchal structure. Additionally, the communication services component ensures the server communicate with its external clients. The administrator in the server side needs to specify an unoccupied communication port for data exchange with the clients. Once the server is started, system states such as server start /stop, and collaborator's $\log$ in/off etc. are reported in an event window. Based on these transparent features, the administrator can effectively manage the server and monitor its working situations.

\section{Client Side Application}

The client side application builds the client tier modules of 4D builder, communication services, and event hub. It enables multidisciplinary planners to conduct planning work in their local systems while communicating and collaborating with each other. Among these modules, the 4D builder supports planning activities including 3D model analysis, simulation item definition, as well as 4D sequence generation. The communication services module allows planners to communicate with remote collaborators, such as server connection, role registration, text charting etc. The event hub module at a low level undertakes all data transmission to underpin high-level operations from planners.

The client side application integrates the user interfaces of all the modules' components (Figure 8). A communication panel is prepared for the user to connect the server, select a predefined specialty role, and list all online collaborators and their states. An instant messenger is also available for the planner to communicate with other online collaborators. A 3D-based planning environment is created as the 4D player for 3D model browsing and analyzing, 3D elements picking, simulation item 
defining, and 4D simulation player. The loaded 3D model and plan from a local system can fill in the 3D element container and the SIMU item container. They are displayed in the 4D player and the plan browser respectively. The same result can be achieved by retrieving data from the 4D item pool in the server side when planners log in the server.

Applying the 4DX client application for creating a plan, planners can navigate and analyze the 3D model in the 4D player via zoon, pan, and rotate operations. In view of the overlapped nature of 3D elements in the model, a topology displaying control is devised to help planners decompose the 3D model. It is able to control the 3D model's elements, displaying one by one in a bottom-up or top-down order. Planners thus have the opportunity to scrutinize every 3D element of the model. On deciding a task definition, planners can pick up related 3D elements to specify the task via the SIMU item definition panel. The defined task is then synthesized with picked $3 \mathrm{D}$ elements to be a simulation item. The newly created simulation item is automatically sent to the server for integration with other defined simulation items, and then received and recorded in the local SIMU item container. With created simulation items, the 4D player is thus able to detect potential conflicts in the plan by 4D simulation. Leveraging the network support, all these planning activities can be conducted across the network by the collaborative sessions of co-navigate, co-sort, co-plan, co-simulate, and co-talk. Therefore, planners' work is open-ended, communicated, and it is possible to achieve a robust plan.

Through a user-centered design, the user interface/interaction (UI) design of the 4DX client is further adapted to be collaboration oriented (Figure 9). In this circumstance, some graphical user interfaces (GUIs), such as the instant messenger and logging panel, are removed or hidden. The planners hence can focus on the main planning tasks independently or collaboratively depending on whether or not they involve online collaborators. In case of no other online planners selected or available, the planner can perform planning individually through the operations of navigate, sort, plan and simulate. In case of selecting online planners, these operations become available in the collaborative sessions of co-navigate, co-sort, co-plan and co-simulate. This flexibility enables 4DX client to be applicable for both offline and online conditions. 


\section{System Validation}

The system validation was performed in a truly geographically distributed condition. Four planners were involved to apply 4DX clients in different locations to collaborate with each other simultaneously across the Internet. A commercially available multiparty video conferencing system named ooVoo was leveraged to bridge the gap among these geographically dispersed planners, who could communicate with each other face-to-face in real time during the planning process. The validation applied several multimedia PCs with dual monitors and connections to the Internet. The dual-monitor setting was dedicated to displaying both 4DX and ooVoo in one client. A prepared entitybased 3D building model (Figure 9) was adopted in the validation. According to the availability of building elements in the 3D model, four roles were correspondingly defined as structure planner, window planner, door planner, and glass planner in the server side. During the testing process, the team members located in different buildings within the University of Wolverhampton, which provided unlimited wireless Internet access for all users.

\section{Process and Result}

The overall validation process followed a serial workflow of co-sort and co-plan. In the cosort session, team members engaged in sorting building information concurrently to customize their specialty-oriented planning context. Subsequently, the team members performed concurrent planning in their own contexts. During these two procedures, co-navigate was utilized to help the plan team analyze the $3 \mathrm{D}$ model while co-talk provided communication services using the ooVoo throughout the whole process. Co-simulate contributed to the co-plan when the plan team wanted to detect potential conflicts during the planning. Both co-navigate and co-simulate were used by the team for concurrent individual work in client sides and collaborative teamwork across the Internet.

When starting the validation planning, all team members signed in the ooVoo first. The server administrator started the 4DX server, and sent the server IP address as well as a communication port to other collaborators via the ooVoo. All the team members then logged in the server and retrieved the 3D model from the server to their local systems. The control tester initiated the co-sort so that all 
collaborators could pick up their 3D elements from the 3D building model. A set of filters for controlling 3D element display state was designed in the UI so that collaborators could use them to filter out relevant 3D building information and obtain their own PBS elements. Taking the advantages of created building information visualization by the system as well as real-time multiparty video conferencing from ooVoo, all planners successfully sort their own 3D elements collaboratively and concurrently across the Internet. Its result is illustrated in Figure 10. As indicated in the illustration, the $3 \mathrm{D}$ elements with black mesh in the model are readable whilst the $3 \mathrm{D}$ elements in the grey solid state are writable for defining simulation items.

After obtaining their own 3D building elements, the collaborators proceeded to the co-plan to conduct planning together. They focused on their own 3D elements to define simulation items. During this procedure, the collaborators still maintained social interaction via ooVoo to exchange planning strategy, adjust their own definition, and give suggestions to other collaborators simultaneously. As they shared the same plan context, updates by any collaborator were reflected on the client machines of other users via server broadcasting in real time. Co-simulate was also conducted in the co-plan process across the Internet so that to identify and eliminate potential conflicts in the co-created plan. A robust plan therefore could be guaranteed under the monitoring from collaborators. The screen shot of this coplan working is illustrated in Figure 11.

The final construction plan that was generated was shown in a hierarchal structure, and could be exported into MS Project to gain the same structured plan with a bar chart (Figure 12). This result kept consistency with that of the verification test. Its complete 4D simulation sequence is presented in Figure 13. These results indicate the effectiveness of the proposed 4D groupware, and further verify the applicability of the interactive definition method. Combining the commercial multiparty video conferencing system of ooVoo, the proposed 4D groupware solution is feasible for geographically dispersed planners to collaboratively conduct planning and simulation across the Internet. 


\section{Findings in Validation}

The validation confirms that the proposed 4D groupware solution is applicable for distributed collaborative planning through teamwork. Applying the server-client mode, geographically dispersed planners can collaborate with each other from their own clients connected by the server. The devised functional modules effectively supported multidisciplinary planners' CSCW sessions across the Internet. They interactively held collaborative sessions during their concurrent planning. This suggests that the underlying real-time data exchange protocols and data flow in the CSCW design were successful to underpin diverse data transmission for team-based collaborative planning. The design of the $4 \mathrm{D}$ item pool in the server side demonstrated feasibility and flexibleness to use a file-based structure to supply the clients with collaborative information. Particularly, the mechanism of roleattribute reactor enables multidisciplinary planners to perform interactive collaboration targeting a unique 3D model even without building information. This feature brings convenience for collaborators to filter out their interested building components which may not belong to BIM.

The use of 3D BIM model will be an important compensation for the proposed solution. The proposed solution aims at a 3D CAD model condition because the entity based 3D CAD model is still prevalent in the construction industry. Since the entity-based 3D CAD model has no building information, a 4D item pool (acting as a central building information repository) is populated by applying several separate files, which supply necessary information for planning. Such a loose information management is flexible to customize collaborative information for different situations. Nevertheless, it increases management burden in the server. A BIM-based file structure such as Industry Foundation Classes (IFC) for the 4D item pool is possible to integrate all parts into a complete information repository. The benefit of adopting BIM into the system lies not only in simplifying the server management, but also possibly automating PBS filter out rather than interactive co-sort in the client side. As BIM-based file has already owned classified building information, collaborators could directly obtain desired PBS information from the server. However, it is reasonable to believe that 3D CAD model and BIM model will always co-exist in the AEC industry. In some circumstances, 3D CAD models are useful to simply represent some temporary elements, e.g. formwork or scaffolding, 
which may not necessarily use BIM. Combining interactive co-sort with automated filtering can be effective and convenient for users to deal with both situations of 3D CAD and BIM models.

The concurrency feature of the prototype needs to be reinforced. A requirement for the collaborators in the validation is that their defined plan tasks comply with an ideal top-down order according to their start time. It needs the planners to define their tasks one by one according to the real temporal and logical construction sequences. This collaborative planning strategy is positive to gain a robust plan and helpful to expose and identify potential conflicts in the 4D simulation. It can also be applicable for real project practice. However, planners are able to define their plan tasks freely and concurrently once obtaining their own 3D elements in normal situations. This freedom and concurrency may cause some earlier tasks to be defined later because of time difference in concurrent work. The 4D simulation of this study excludes this possibility for the purpose of controlling the validation experiment. In a real situation, this exclusion can cause later defined tasks with early start time to be neglected in the simulation. In order to satisfy the concurrent operation's need, the implemented 4D simulation generation mechanism needs modification to become fully time oriented. The remedy of this pitfall would be positive for this groupware to suit concurrent planning situation.

The dual-monitor configuration for the prototype provided convenience in the validation. Because of using multiple applications of the 4DX and the ooVoo at the same time, a clear and ease-ofuse arrangement for the system is important to achieve good usability. Real-time collaborative 4D planning has high demands for user tasks' performance including 3D operation, interactive definition, and social interaction. These mixed user tasks ask for suitable operation schemes to support user's task performance. Dual-monitor configuration permits users to easily conduct main task operations instead of frequently switching user interfaces among different applications. It thus can be helpful to improve the usability of the whole system. Interestingly, it is an attractive topic regarding the interaction design for the promotion of user performance in the condition of multiple monitors and applications. Some research efforts have been invested into this human factor field. It is anticipated combing related achievements with current distributed 4D groupware for further study. 
Finally the validation further verifies the applicability of the interactive definition method for collaborative 4D planning in a truly geographically distributed condition. The validation result shows that geographically dispersed multidisciplinary planners are able to co-create a robust plan via the team worked social interaction, user-system interaction, and plan data incorporation in an open shared social-technical context. The use of 3D model for creating the social-technical context is positive to foster collaborative planning and caters for the planner's mental process. Within this context, the planners are motivated to ponder planning strategies, exchange information with collaborators, and formulate solutions to define simulation items. Meanwhile, the application of commercially available tool of ooVoo is helpful to achieve social interaction. As the interactive definition method permits the planners to gain external information inputs from collaborators during planning, its 4D planning process hence features open-ended, communicated, and collaborative characteristics. Additionally, the co-created construction plan and generated 4D simulation in the validation is evidence that the use of simulation item parameters provided by the method is effective to define $4 \mathrm{D}$ construction plan. They show convenience and efficiency for network transmission, plan synthesis, and simulation generation.

\section{Conclusion and Future Work}

This paper proposed a model-based groupware solution that was validated to be effective for distributed real time collaboration. Its collaborative mechanisms, including software architecture, application mode and data flow, as well as data exchange protocol, are applicable for synchronous model-based teamwork environments. Applying the proposed 4D groupware, multidisciplinary planners can effectively perform collaborative planning across the boundaries of time, location and specialty. The applicability of collaborative mechanisms in the proposed groupware can be useful for equivalent applications in the AEC industry and other industries. Given the social-technical context for collaborative planning, paying attention to only technical aspects such as data integration and interoperability is constructive in groupware development but insufficient to guarantee collaborative teamwork when applying it. This study takes both social and technical issues into account to explore a successful 4D groupware development. Although its validation was performed in a simulated construction project, it still highlighted essential issues of applying the 4D groupware in a distributed condition. The validation also revealed the need for future functionality that will enhance CSCW 
design, social coordination, awareness, and user interface/interaction design. The discussion of these sort of issues is out of the scope of this paper, and be expected a further clarification in another report. The demonstrated benefits and exposed weaknesses in the current solution will be positive to nurture more practical $4 \mathrm{D}$ groupware solutions. It is meaningful to turn construction planning from a multidisciplinary activity to be an interdisciplinary practice. Its industry project validation will further verify its effectiveness, advancement and disclose potential pitfalls. More insights about applying the model-based groupware for distributed collaborative teamwork are expected to be gained in the future.

\section{References}

Autodesk (2010). Revit architecture 2011 user's guide, Autodesk, Inc. April 2010.

Bentley (2011). Dynamic collaboration for iterative project review, Bentley Systems, Inc. Accessible online: http://www.bentley.com/en-US/Products/ProjectWise+Navigator/i-model.htm

Collier, E. and Fischer, M. (1996). Visual-based scheduling: 4D modelling on the San Mateo County Health Centre, Proceedings of the 3rd ASCE Congress on Computing in Civil Engineering, Anaheim, CA.

Dawood, N., Scott, D., Sriprasert, E., and Mallasi, Z. (2005). The virtual construction site (VIRCON) tools: An industrial evaluation, ITcon Vol. 10, Special Issue From 3D to nD modelling , pg. 43-54, http://www.itcon.org/2005/5

Fischer,G., Giaccardi, E., Eden, H., Sugimoto, M., Ye, Y. (2005). Beyond binary choices: Integrating individual and social creativity, International Journal of Human-Computer Studies, Volume 63, Issues 4-5, October 2005, Pages 482-512

Frohlich, B., Fischer, M., Agrawala, M., Beers, A., and Hanrahan, P. (1997). Collaborative production modelling and planning. IEEE Computer Graphics and Applications, 17(4), 13-15. 
Fruchter, R. (1999). Architecture/Engineering/Construction Teamwork: A Collaborative Design and Learning Space, Journal of Computing in Civil Engineering, October 1999, Vol 13 No.4, pp 261-270.

Garner, S. and Mann, P. (2003). Interdisciplinarity: perceptions of the value of computer-supported collaborative work in design for the built environment, Automation in Construction, Volume 12, Issue 5, September 2003, Pages 495-499

Gtwiki (2009). SVN Client setup, gtwiki, March 2009. Accessible online: http://www.gtwiki.org/mwiki/index.php?title=SVN_Client_setup

Greenberg, S. (1991). Computer-supported cooperative work and groupware. International Journal of Man-Machine Studies, Volume 34, Issue 2(February 1991), ISSN: 0020-7373, Academic Press Ltd. London, UK

Goldstein, H. (2001). 4D: Science fiction or virtual reality? Construction.com Features, 16 April, available at http://www.bal4.com/news/2001-04-16-construction.pdf, accessed 20 July 2010.

Golparvar-Fard, M., Peña-Mora, F., Arboleda, C. A., and Lee, S. (2009).Visualization of Construction Progress Monitoring with 4D Simulation Model Overlaid on Time-Lapsed Photographs, Journal of Computing in Civil Engineering, Vol. 23, No. 6, November 1, 2009. CASCE, ISSN0887-3801/2009/6$391-404 / \$ 25.00$.

Grudin, J. (1994). Groupware and social dynamics: eight challenges for developers, Communications of the ACM archive, Volume 37, Issue 1 (January 1994), pp92 - 105, 1994, ISSN: 0001-0782

Heesom, D. and Mahdjoubi, L. (2004). Trends of 4D CAD applications for construction planning, Construction Management \& Economics, 22, 171-182. 2004. 
Hendrickson, C. (1998). Project Management for Construction, Department of Civil and Environmental Engineering, Carnegie Mellon University, Pittsburgh, PA 15213. Available from:

$\underline{\text { http://www.ce.cmu.edu/pmbook/ }}$

Jongeling, R. and Olofsson, T. (2006). A method for planning of work-flow by combined use of location-based scheduling and 4D CAD, Automation in Construction, In Press, Corrected Proof, Available online 24 May.

Kang, J., H., Anderson, S., D., and Clayton, M., J. (2007). Empirical Study on the Merit of Web-Based 4D Visualization in Collaborative Construction Planning and Scheduling, Journal of Construction Engineering and Management. Volume 133, Issue 6, pp. 447-461, 2007

Khanzode, A., Fischer, M., Reed, D. (2008). Benefits and lessons learned of implementing building virtual design and construction (VDC) technologies for coordination of mechanical, electrical, and plumbing (MEP) systems on a large healthcare project, ITcon Vol. 13, Special Issue Case studies of BIM use, pg. 324-342, http://www.itcon.org/2008/22

Kim, C., Kim, H., Park, T., and Kim, M. K. (2010). Applicability of 4D CAD in Civil Engineering Construction: Case Study of a Cable-Stayed Bridge Project, Journal of Computing in Civil Engineering. 2010. doi:10.1061/(ASCE)CP.1943-5487.0000074

Koo, B. and Fischer, M. (2000). Feasibility Study of 4D CAD in Commercial Construction, Journal of Construction Engineering and Management Jul/Aug

Ku. K., Pollalis, S., Fischer, M., Shelden, D. (2008). 3D model-based collaboration in design development and construction of complex shaped buildings, ITcon Vol. 13, Special Issue Case studies of BIM use, pg. 258-285, http://www.itcon.org/2008/19

Li, H., Ma, Z., Shen, Q., Kong, S. (2003). Virtual experiment of innovative construction operations. Automation in Construction, 12(5): 561-575. 
Manning, R., Messner, J. (2008). Case studies in BIM implementation for programming of healthcare facilities, ITcon Vol. 13, Special Issue Case studies of BIM use, pg. 246-257, http://www.itcon.org/2008/18

Maxfield, J., Fernando T., Dew P.M., (1998). Synchronous collaboration through a distributed virtual environment in concurrent engineering, presence: teleoperators and virtual environments, pages 241 261, Vol.7, Issue 3.

Mills, K., L. (2003). Computer-Supported Cooperative Work. Encyclopedia of Library and Information Science, Marcel Dekker, Inc., pp 666 - 677, DOI: 10.1081/E-ELIS-120008706

Pazlar, T., Turk, Z. (2008). Interoperability in practice: geometric data exchance using the IFC standard, ITcon Vol. 13, Special Issue Case studies of BIM use, pg. 362-380, http://www.itcon.org/2008/24

Pena-Mora, F., Hussein, K., Vadhavkar, S., Benjamin, K. (2000). CAIRO: a concurrent engineering meeting environment for virtual design teams, Artificial Intelligence in Engineering 14 (2000) 203-219

Shelbourn, M., Bouchlaghem, N.M., Anumba, C. and Carrillo, P. (2007). Planning and implementation of effective collaboration in construction projects, Construction Innovation, Vol. 7 No. 4, 2007, pp. $357-377$.

Sriprasert, E. and Dawood, N. (2003). Multi-constraint information management and visualisation for collaborative planning and control in construction, ITcon Vol. 8, Special Issue eWork and eBusiness, pg. 341-366, http://www.itcon.org/2003/25

Sulankivi, K., Kähkönen, K., Mäkelä, T., Kiviniemi, M. (2010). 4D-BIM for construction safety planning, CIB 2010 World Congress proceedings. Barrett, Peter, Amaratunga, Dilanthi, Haigh, 
Richard, Keraminiyage, Kaushal \& Pathirage, Chaminda (eds). CIB (2010).

http://www.cib2010.org/post/files/papers/1167.pdf , Accessed 17th August 2010

Synchro (2010), Synchro user guide, version 3.1415.1. Synchro Ltd. April 2010.

Tanyer, A., M. and Aouad, G. (2005). Moving beyond the fourth dimension with an IFC-based single project database, Automation in Construction, Volume 14, Issue 1, January 2005, Pages 15-32.

Tekla (2010), Tekla Structures system manual, product version 16.1. Tekla Co., 2010

The University of Salford (2005). nD Modelling Road map, a Vision for nD enabled Construction.

Tse, T. K., Wong, K. A. and Wong, K. F. (2005). The utilisation of building information models in nD modelling: A study of data interfacing and adoption barriers, ITcon Vol. 10, Special Issue From 3D to nD modelling , pg. 85-110, http://www.itcon.org/2005/8

Uher, T. E. and Loosemore, M. (2004). Essentials of Construction Project Management, University of New South Wales Press Ltd.

Vries. B., D. and Broekmaat, M. (2007). Generation of a construction planning from a 3D CAD model, Automation in Construction, Volume 16, Issue 1, January 2007, Pages 13-18

Zhou, W., Heesom, D., Georgakis, P., Nwagboso, C., and Feng, A. (2009). An interactive approach to collaborative 4D construction planning, ITcon Vol. 14, Special Issue Technology Strategies for Collaborative Working, pg. 30-47, http://www.itcon.org/cgi-bin/works/Show?2009 05 


\section{List of Figure Captions}

Figure 1: Rationale of interactive collaboration for 4D construction planning (Zhou et al, 2009)

Figure 2: Scenario of conditional session A and B with other unconditional sessions

Figure 3: Software architecture

Figure 4: Event hub structure

Figure 5: Server/client mode

Figure 6: Data flow between the server and the clients

Figure 7: User interface of 4DX server application

Figure 8: User interface of 4DX client application

Figure 9: Collaboration oriented UI design of 4DX client in online application

Figure 10: Co-sort results of four planners in the validation

Figure 11: Client side dual-monitor screen shot of 4DX and ooVoo in co-plan

Figure 12: Consistent construction plan created by 4DX and outputted into MS Project

Figure 13: Generated 4D simulation sequence in the validation

Table 1: Attribute values of a PBS element for different collaborators

\begin{tabular}{|c|c|c|c|c|}
\hline Situation & Collaborator A & Collaborator B & Collaborator C & Collaborator D \\
\hline $\mathbf{1}$ & $\mathrm{F}$ & $\mathrm{F}$ & $\mathrm{F}$ & $\mathrm{F}$ \\
\hline $\mathbf{2}$ & $\mathrm{W}$ & $\mathrm{R}$ & $\mathrm{R}$ & $\mathrm{R}$ \\
\hline $\mathbf{3}$ & $\mathrm{C}(\mathrm{W})$ & $\mathrm{C}(\mathrm{W})$ & $\mathrm{R}$ & $\mathrm{R}$ \\
\hline
\end{tabular}

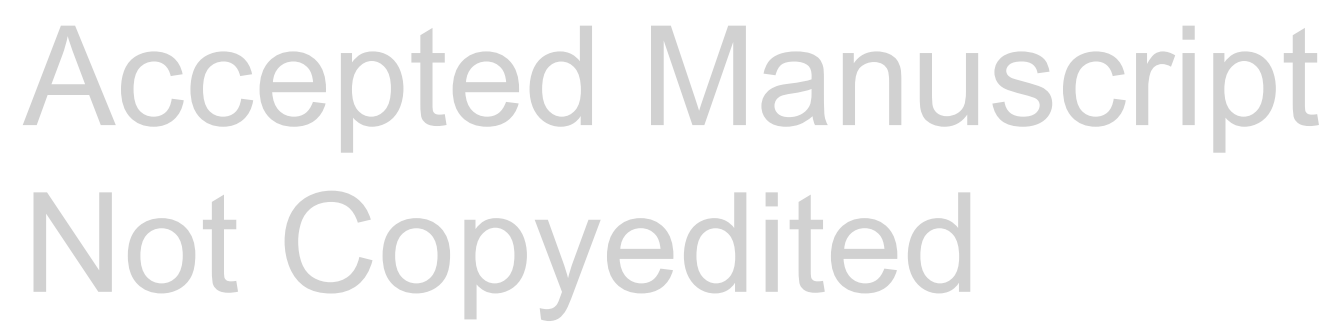


Figure 1:Rationale of interactive collaboration for $4 \mathrm{D} \ldots$

Journal of Computing in Civil Engineering. Submitted August 26, 2010; accepted July 28, 2011; posted ahead of print July 30, 2011. doi:10.1061/(ASCE)CP.1943-5487.0000153

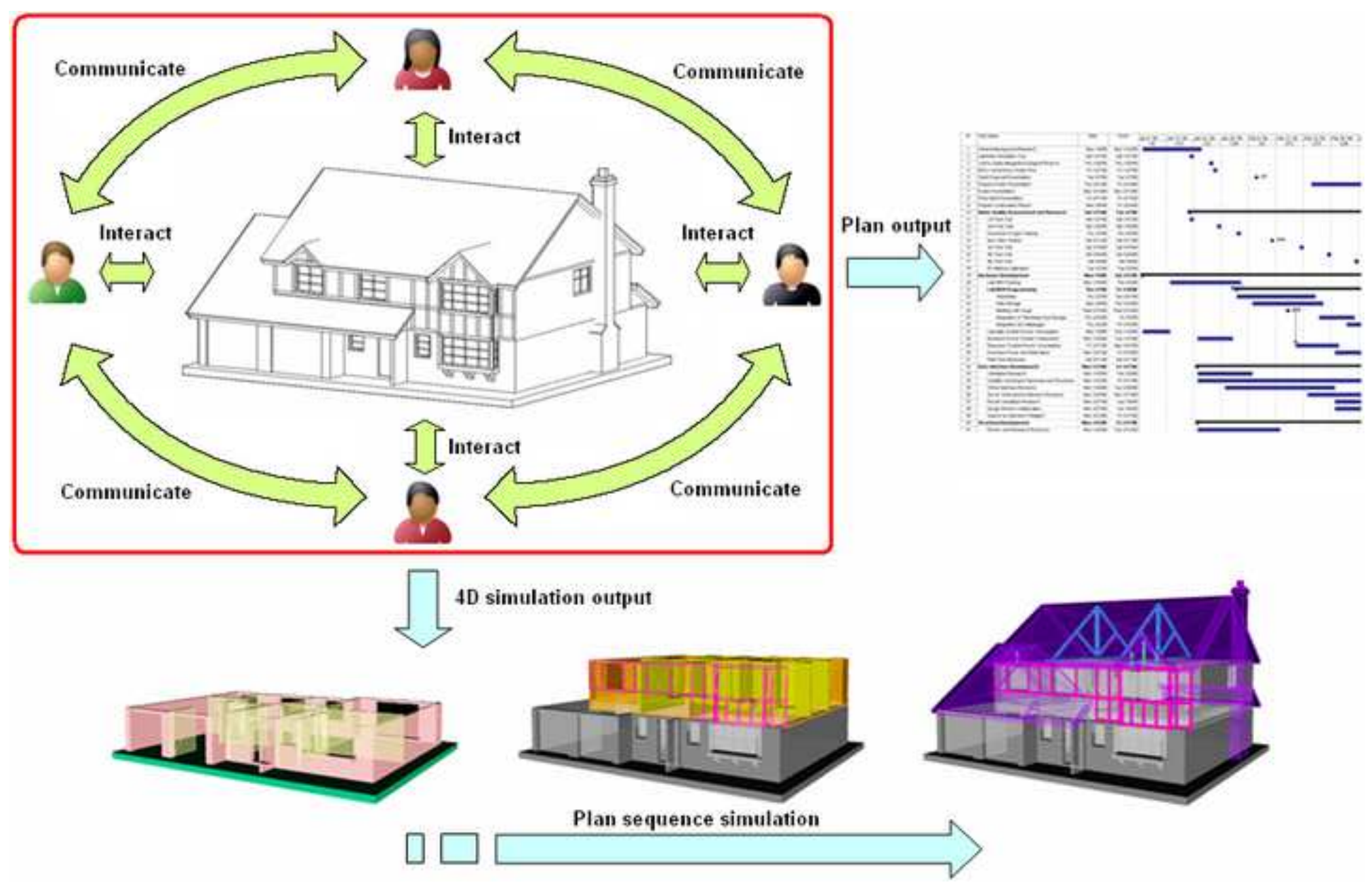

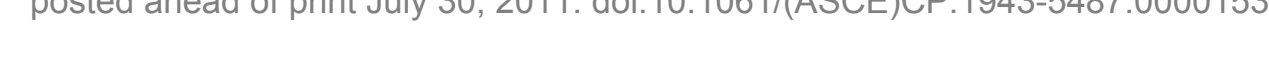


Figure 2: Scenario of conditional session A and B with other ...
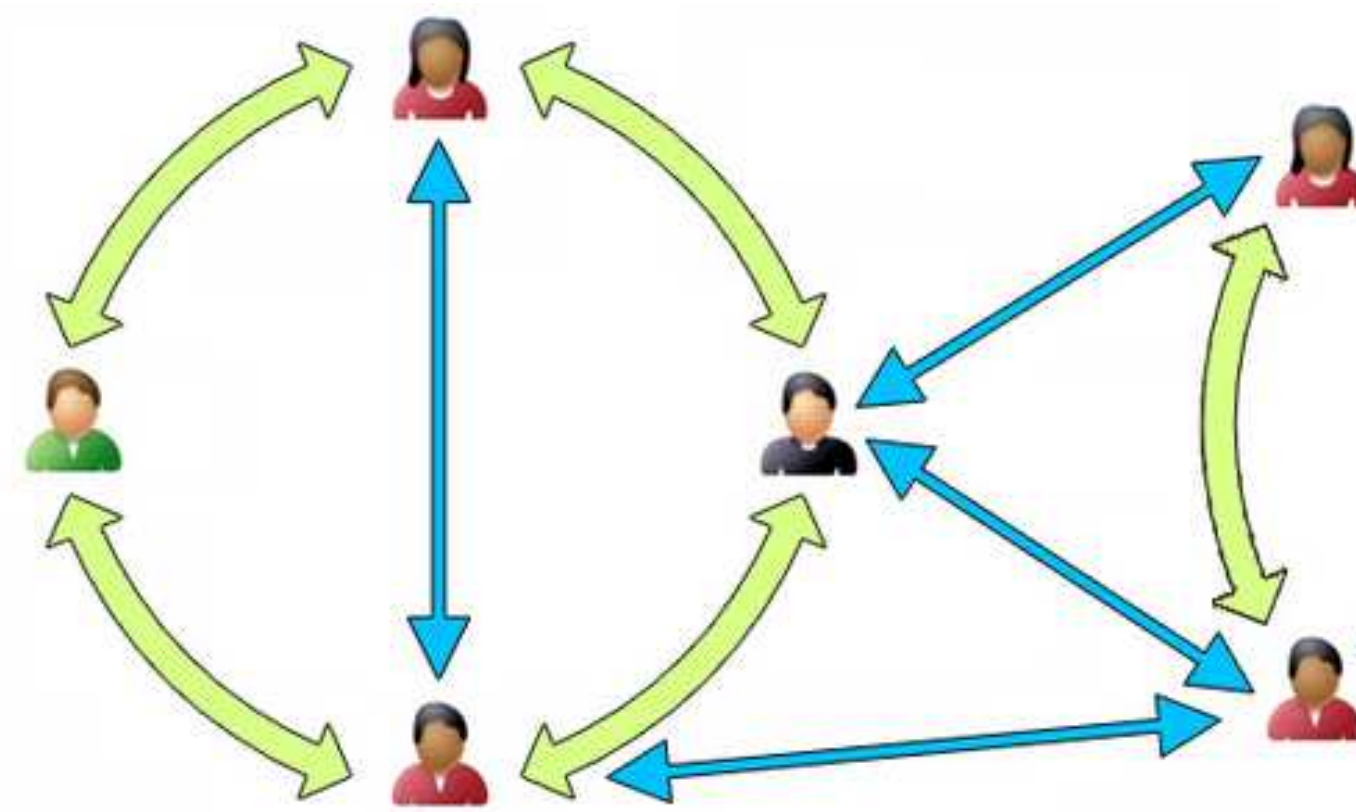

Conditional session $\mathrm{A}$

Conditional session

Uncondtional session 


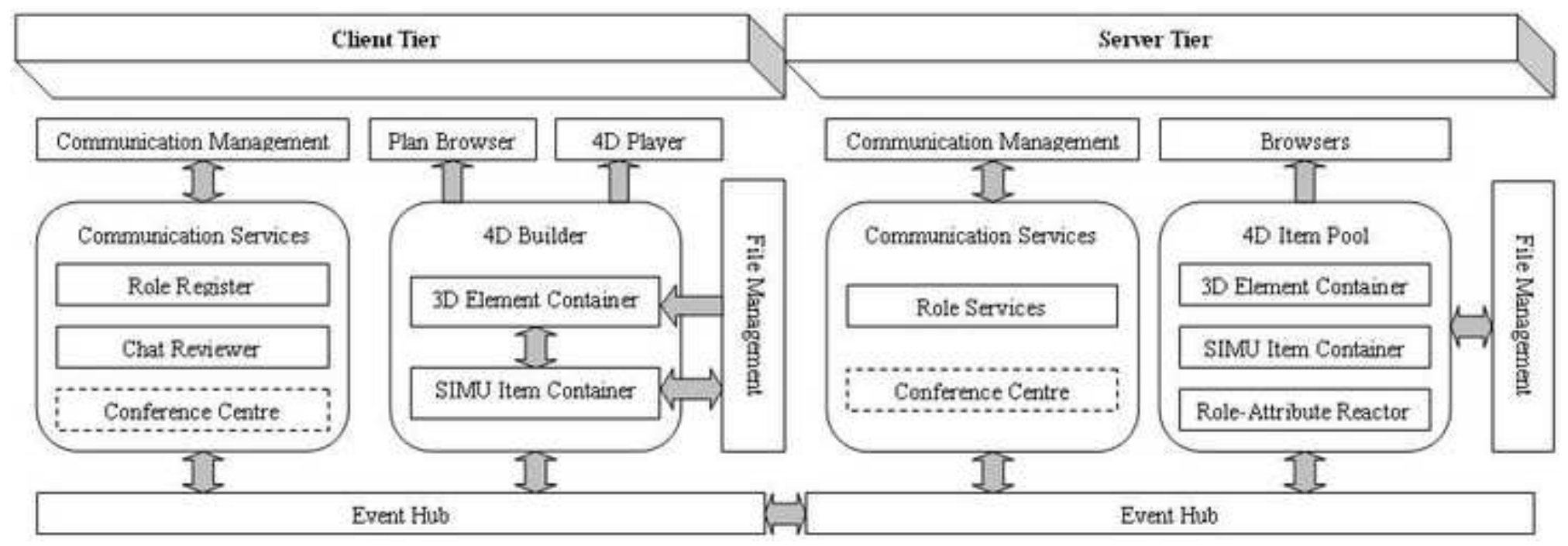


Figure 4: Event hub structure

Journal of Computing in Civil Engineering. Submitted August 26, 2010; accepted July 28, 2011; posted ahead of print July 30, 2011. doi:10.1061/(ASCE)CP.1943-5487.0000153

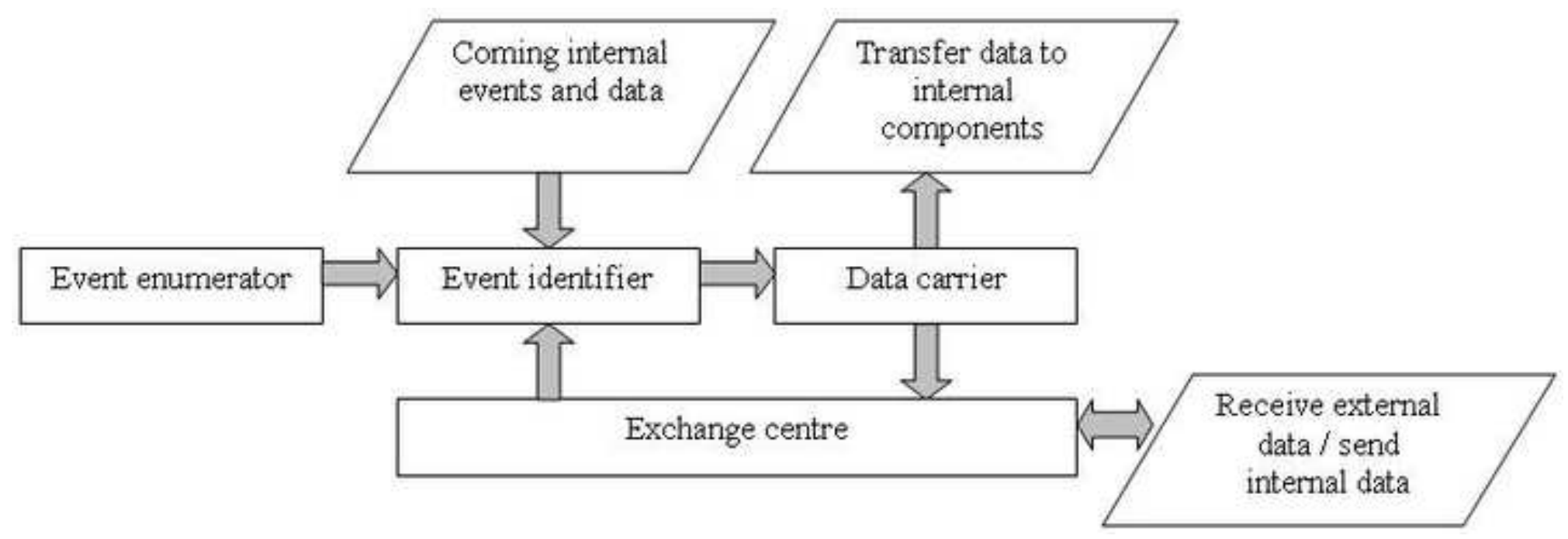




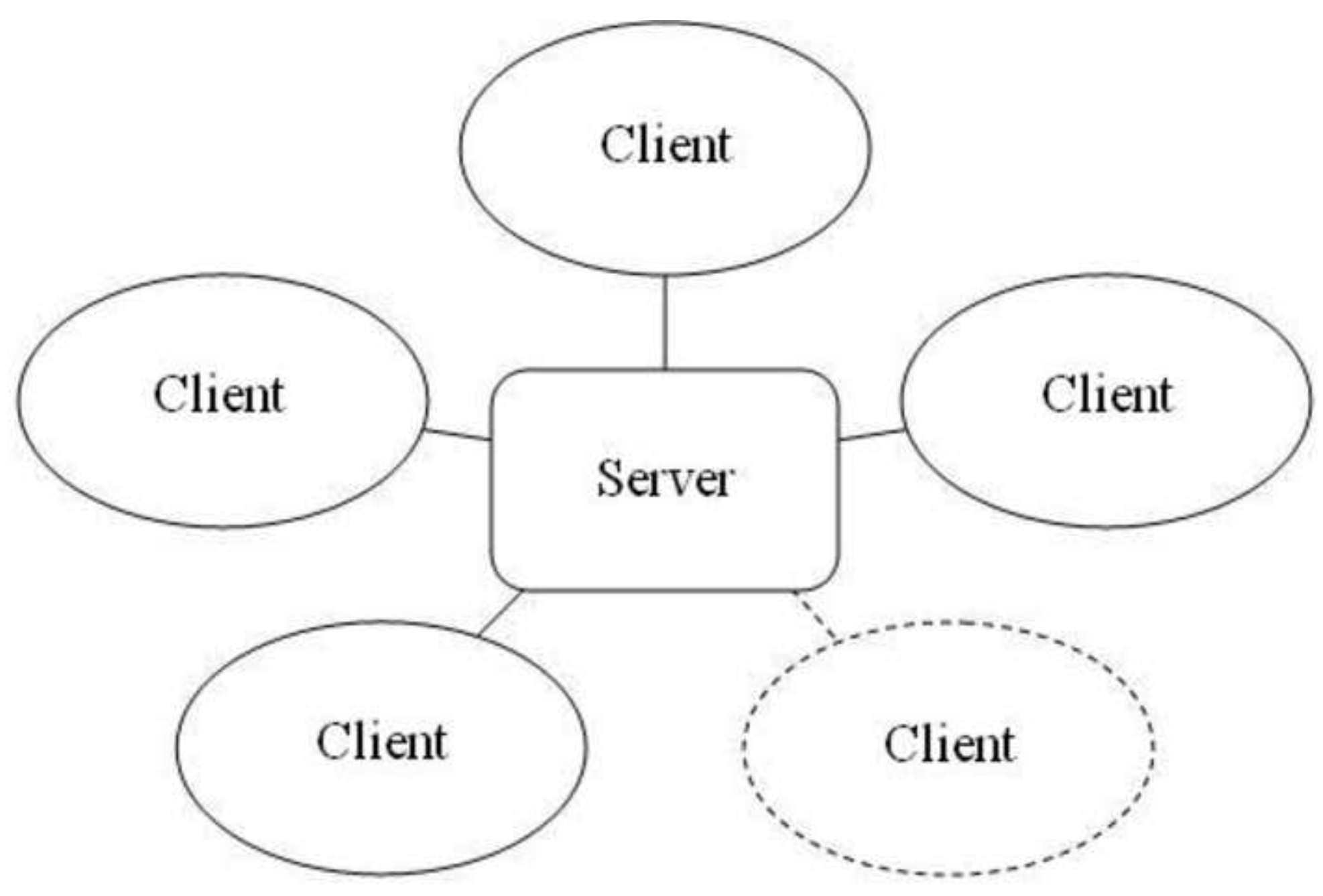




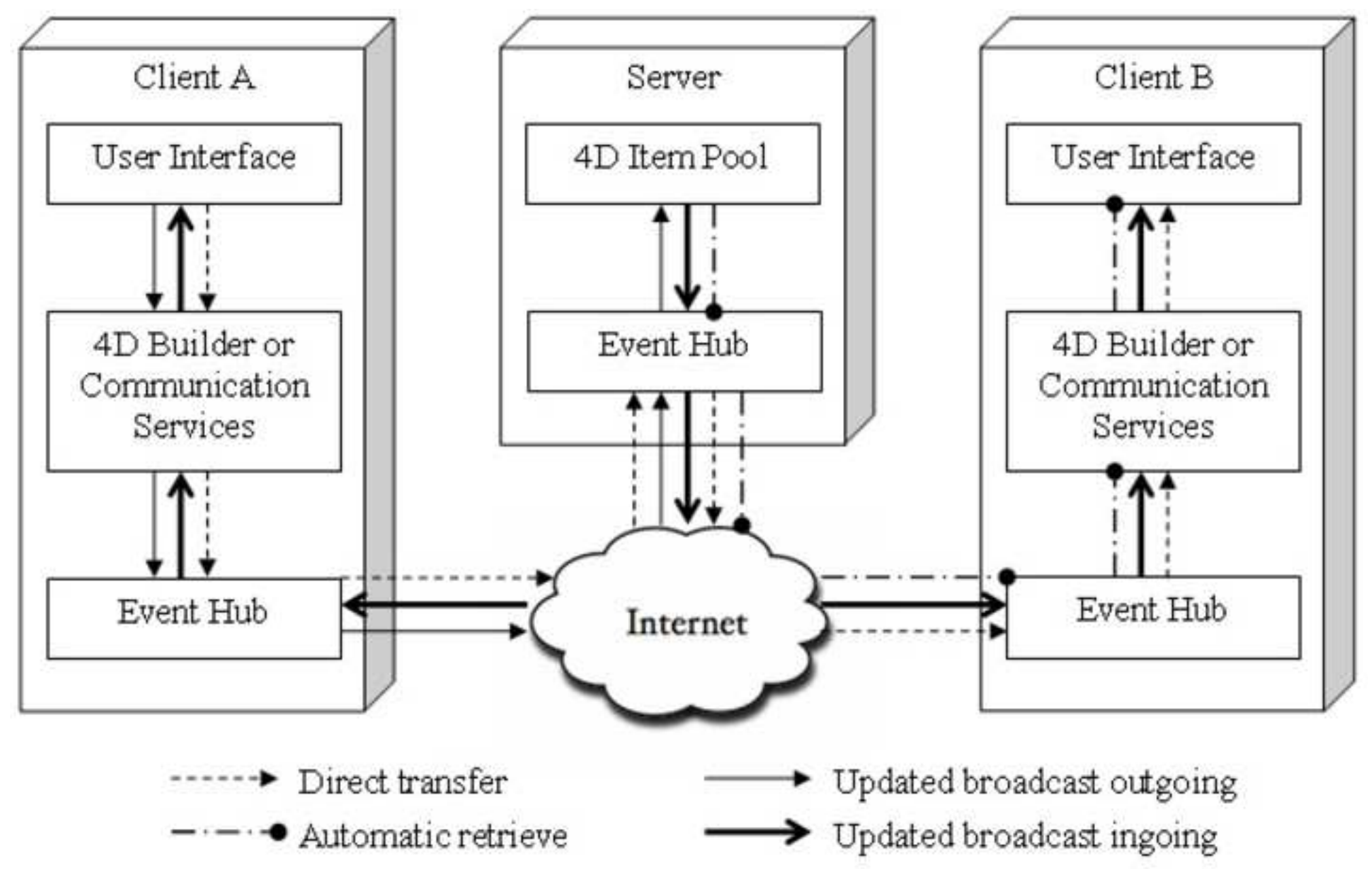


Figure 7: User interface of 4DX server application

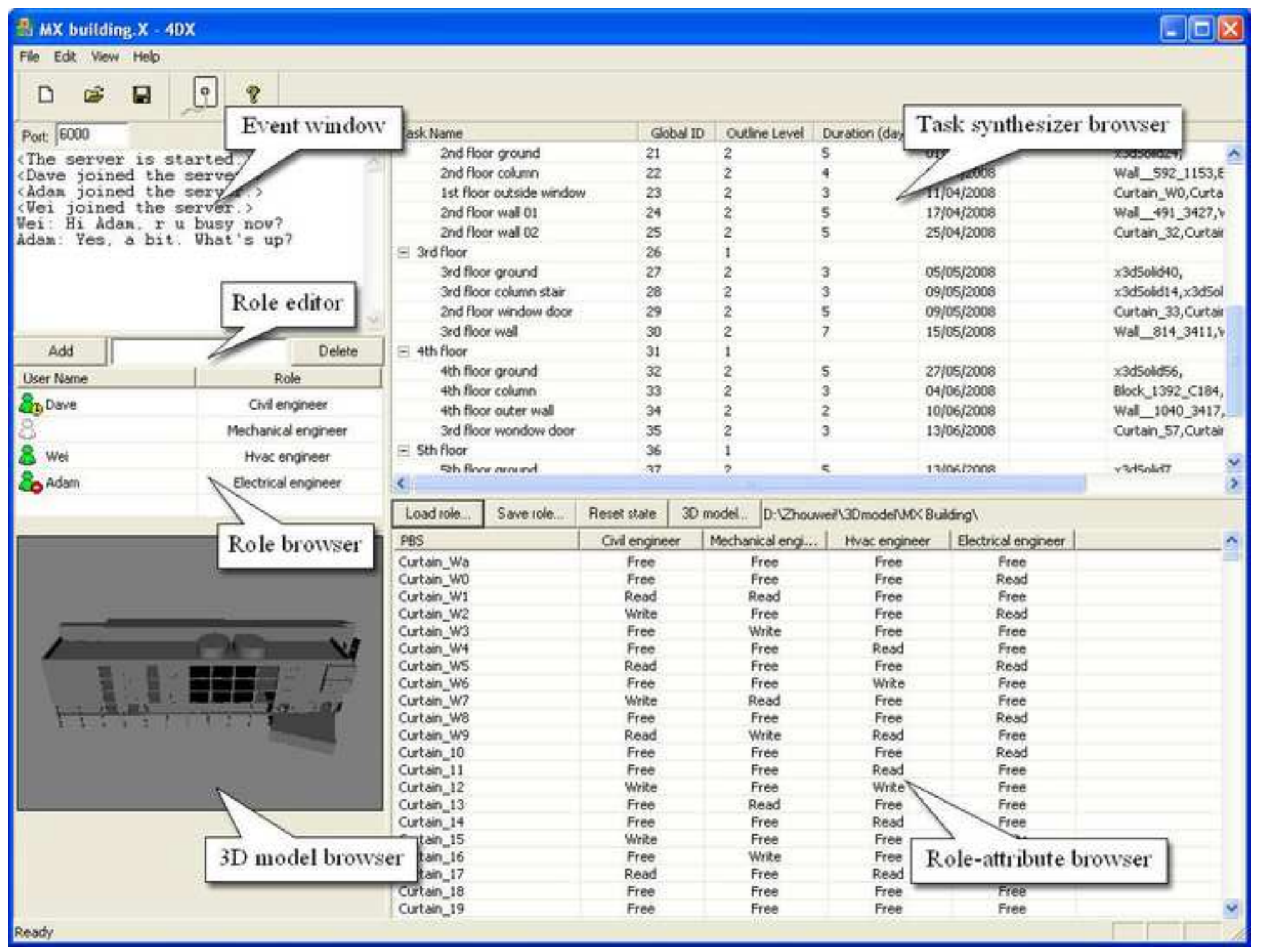


Figure 8: User interface of 4DX client application

Journal of Computing in Civil Engineering. Submitted August 26, 2010; accepted July 28, 2011; posted ahead of print July 30, 2011. doi:10.1061/(ASCE)CP.1943-5487.0000153

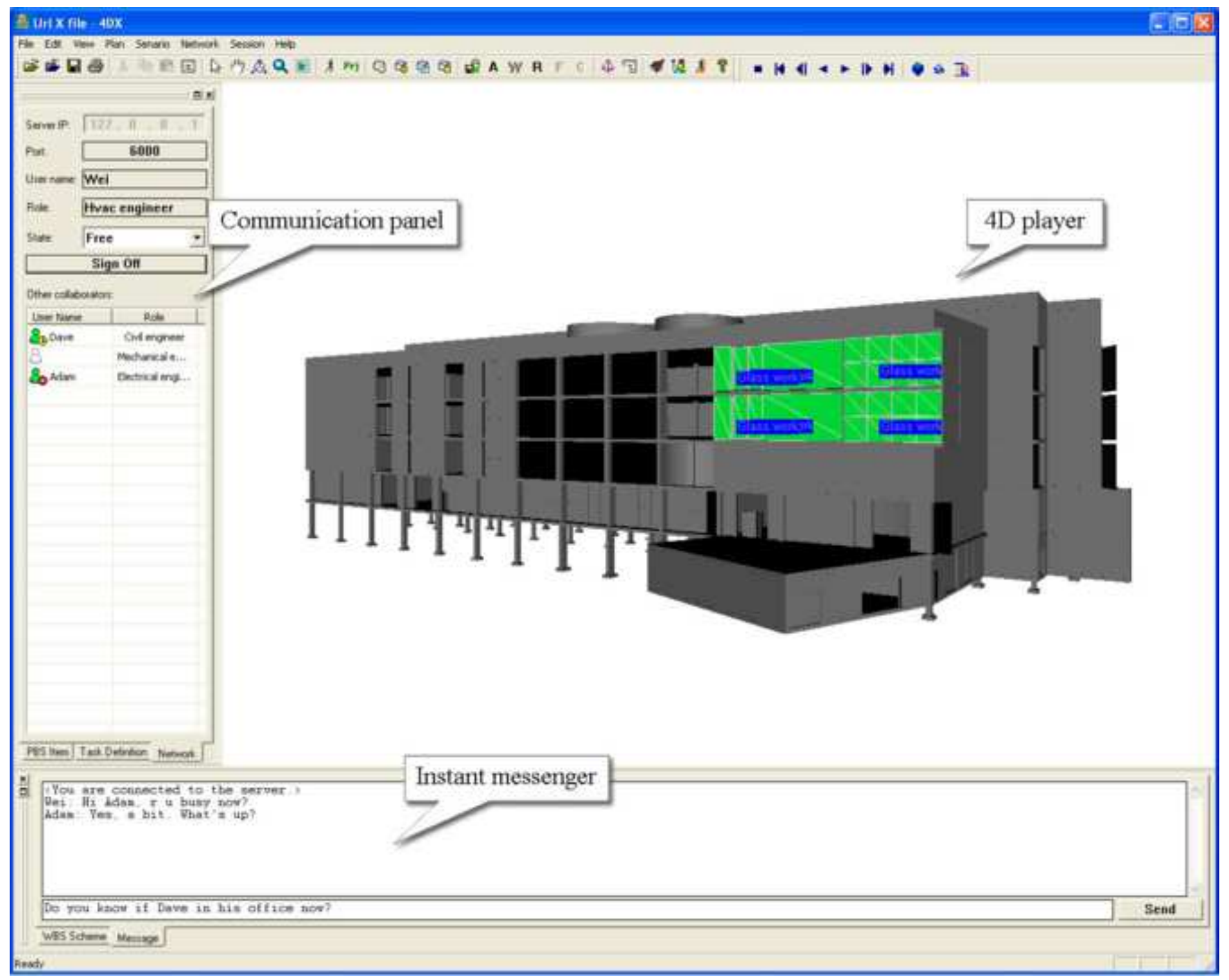

एँ

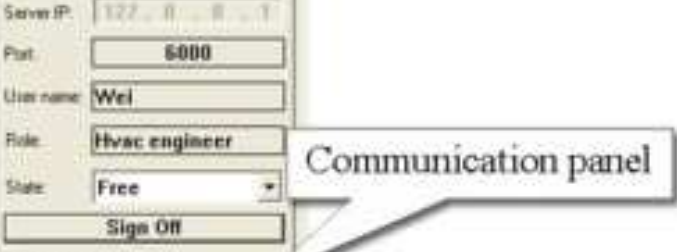

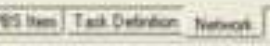

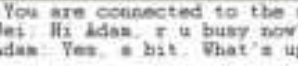

Instant messenger

Dave in his offec and 
Figure 9: Collaboration oriented UI design of 4DX client ...

Journal of Computing in Civil Engineering. Submitted August 26, 2010; accepted July 28, 2011; posted ahead of print July 30, 2011. doi:10.1061/(ASCE)CP.1943-5487.0000153

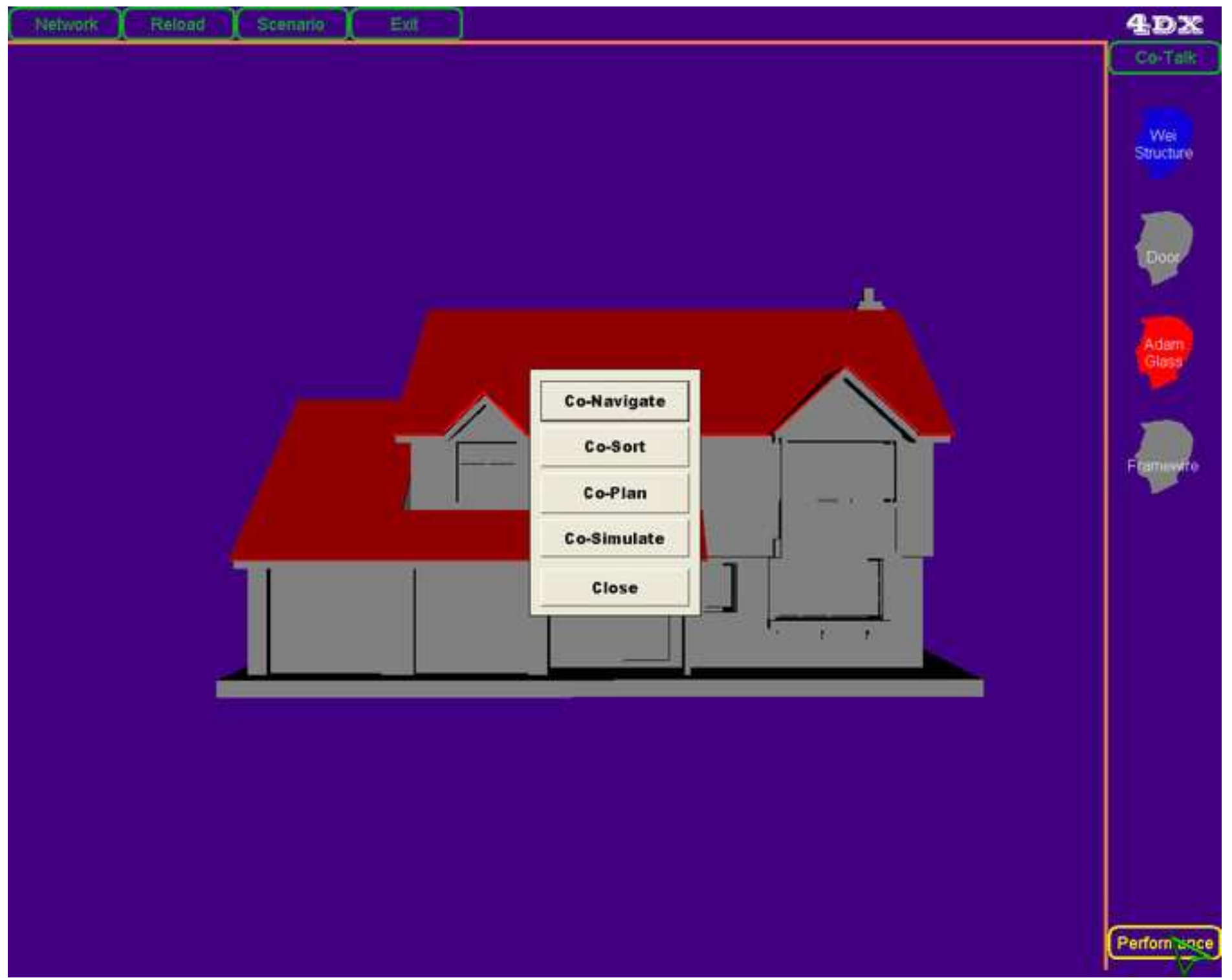




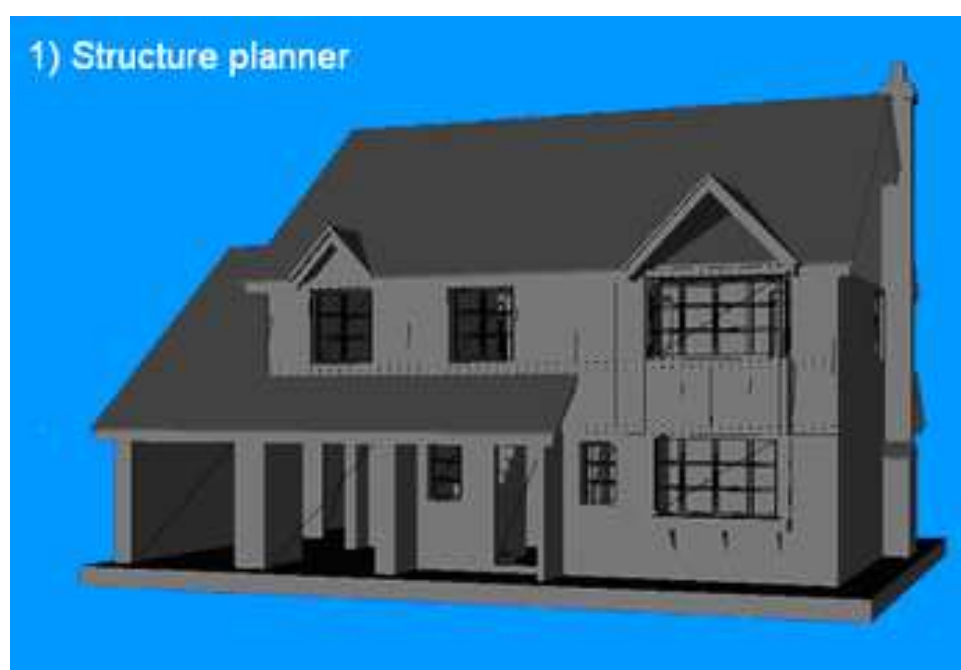

3) Door planner

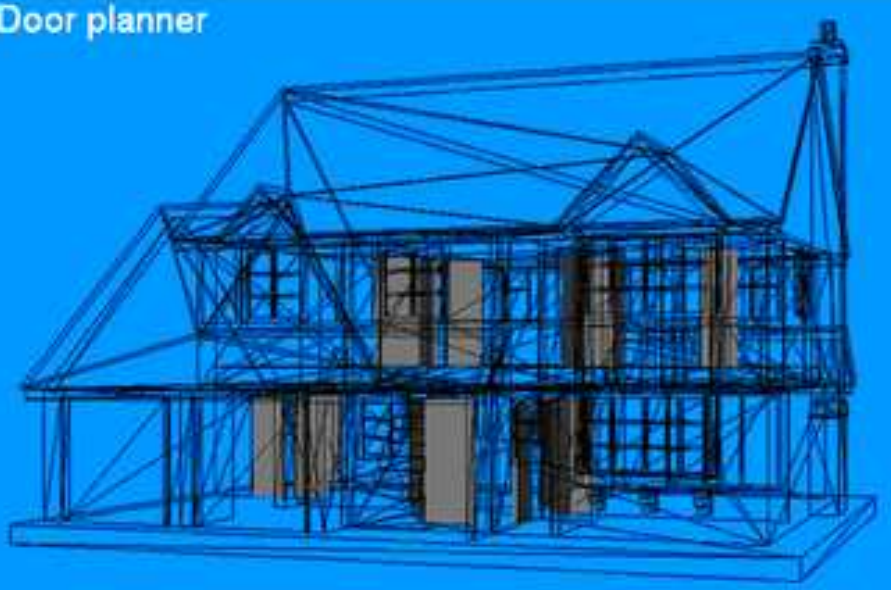

2) Window planner

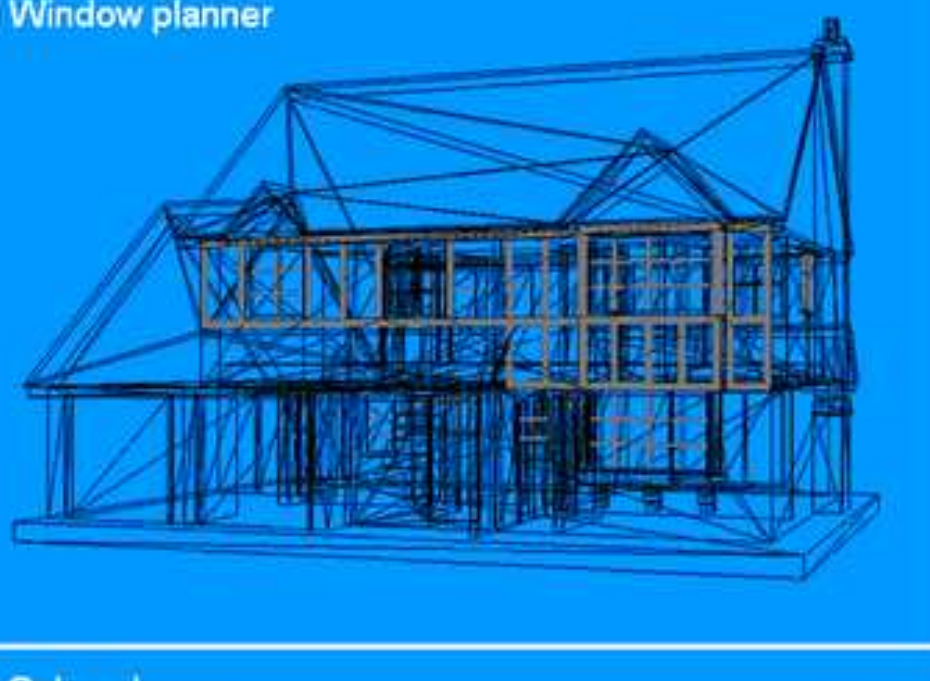

4) Galss planner

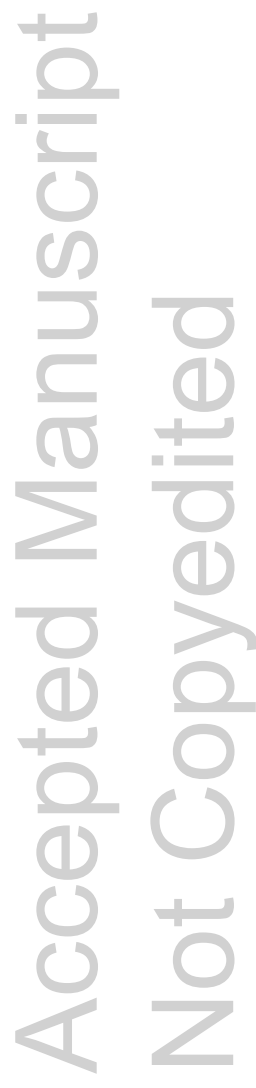



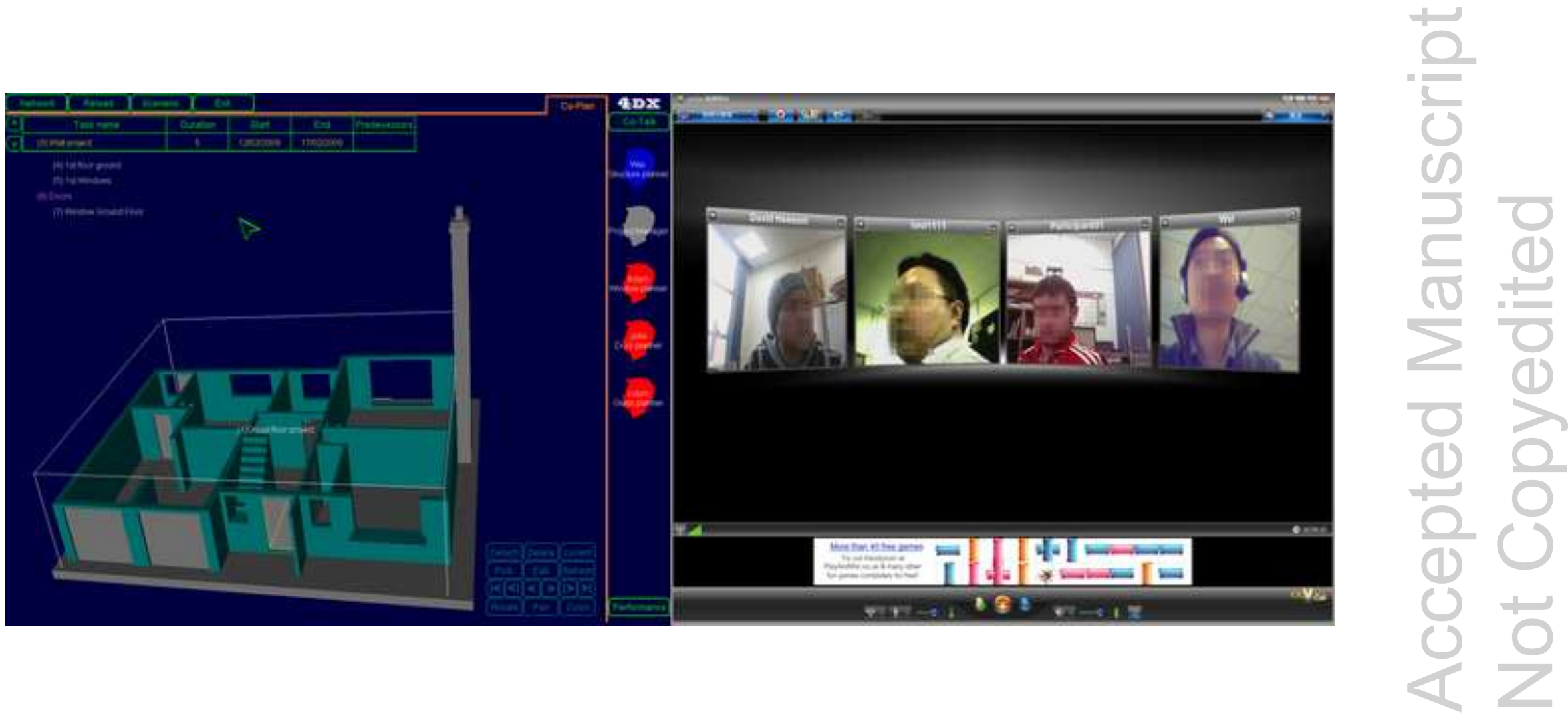
Figure 13: Generated 4D simulation sequence in the validation

Journal of Computing in Civil Engineering. Submitted August 26, 2010; accepted July 28, 2011; posted ahead of print July 30, 2011. doi:10.1061/(ASCE)CP.1943-5487.0000153
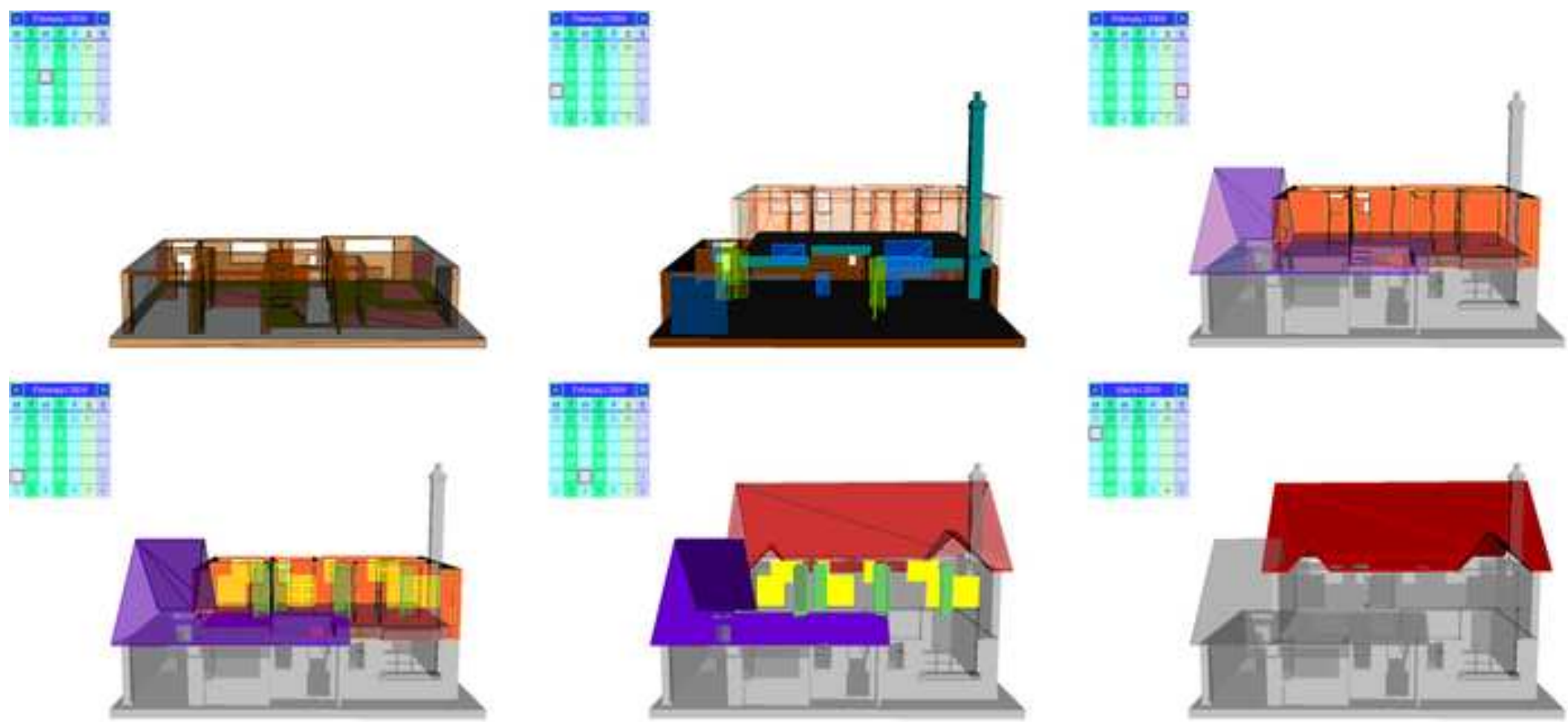